\title{
AN ELECTRICAL DOUBLET THEORY OF THE NATURE OF THE MOLECULAR FORCES OF CHEMICAL AND PHYSICAL INTERACTION.
}

\author{
By R. D. KLEeman.
}

SYNOPSIS.

\begin{abstract}
Electrical doublet theory of molecular attraction.-The author has previously found from data on the internal heat of evaporation of liquids that the attraction between two atoms is proportional to the product of the square roots of their atomic weights. It is now suggested that a neutral atom with a positive nucleus surrounded by electrons is an electrical doublet; and it is shown that the force between two such doublets would on the average be an attraction proportional to the product of the moments of the two doublets. This result combined with the previous one leads to the conclusion that the electrical moment of an atom is proportional to the square root of its atomic weight. Similarly the electrical moment of a molecule comes out equal to the sum of the moments of the component atoms. This theory of molecular attraction is supported by the fact that the stopping power of gases for alpha rays is known to be proportional to the sum of the square roots of the atomic weights of the component atoms; for this law follows directly if we suppose the transfer of energy from the alpha particles to the gas molecules occurs solely as a result of electrical forces exerted by the charged rays on the molecular doublets.
\end{abstract}

Previous Investigations of the Molecular Forces

I T will be instructive and useful to consider as an introduction to the subject of this paper the nature of the law of force between two molecules which can be deduced directly from physical data. I have shown ${ }^{1}$ that the force of attraction between two molecules deduced from data on the internal heat of evaporation of liquids, is given by the expression

$$
\frac{\phi\left(\frac{T}{T_{c}}, \frac{z}{x_{c}}\right)}{z^{5}} \cdot(\Sigma \sqrt{a})^{2},
$$

where $z$ denotes the distance of separation of the two molecules in the liquid whose temperature is $T, x_{c}$ the average distance of separation of molecules of the liquid at the critical point whose critical temperature is $T_{c}, \Sigma \sqrt{a}$ denotes the sum of the square roots of the atoms of a molecule, and $\phi\left(T / T_{c}, z / x_{c}\right)$ is a function whose form is left arbitrary by the data, and which for shortness will be denoted by $(\phi)$. It appears from the foregoing expression that the attraction between two molecules is a

${ }^{1}$ Phil. Magazine, May, I910, pp. 783-809; Jan., I9II, pp. 83-102. Proc. Camb. Phil. Soc., XVI., Pt. 7, pp. 583-598. 
function of their temperature as well as of their distance of separation. We would expect, of course, that the attraction between two molecules might depend on the temperature, since a change in temperature is accompanied by a change in the internal molecular energy, which probably involves a change in atomic configuration, as is shown by the positive value of the specific heat. The foregoing law, it should be remarked, was obtained from latent heat data taking into account the following mathematical theorem: The nature of a function $F$ of two independent variables $x$ and $y$ can be determined approximately in a definite manner only from data giving the values of the function corresponding to variations of each of the variables keeping the other constant, while if the form of the function is determined from data corresponding to the simultaneous variation of $x$ and $y$ it will contain an arbitrary function. A corollary of this theorem is, if the assumption is made that the function contains one variable only, and a form of the function is found agreeing with the facts, this does not signify that the assumption made is true. Now the density of a liquid changes on change of temperature, and the internal heat of evaporation is therefore a function of the two independent variables temperature and distance of separation of the molecules, which vary simultaneously. The law deduced from such data by itself should therefore contain an arbitrary function, and the nature of this function must accordingly be determined by considering in addition data relating to other quantities. This has been carried out by the writer ${ }^{1}$ but the results need not concern us here, except that the function varies little with $z$ and $T$. For our present purpose it is important to notice that the function involves quantities depending on the critical state of the substance to which it refers, which indicates that the nature and magnitude of the attraction between two molecules in the substance is influenced by the surrounding molecules, as we might expect.

The Molecular Forces are Explained by Considering the Atous as Electrical Doublets.

In this paper an attempt will be made to explain the nature of the molecular attraction under consideration (which give rise to the internal heat of evaporization of a liquid, its surface tension, intrinsic pressure, chemical interaction between its atoms and molecules, etc.) by the electric charges each atom is supposed to contain. It will be of interest and serve as an introduction to the main investigation, to consider the attraction which one of the electrical charges in an atom would exert on another atom, supposing that the latter atom behaves as a spherical

${ }^{1}$ Loc. cit. 
conductor of electricity. The attraction in that case is given by the well known expression

$$
\frac{e^{2} r_{1}^{3}\left(2 f^{2}-r_{1}^{2}\right)}{f^{3}\left(f^{2}-r_{1}^{2}\right)^{2}},
$$

where $e$ denote the electric charge in the former atom, $r_{1}$ the radius of the latter atom, and $f$ the distance between their centers. When $r_{1}{ }^{2}$ is small in comparison with $f^{2}$ this expression reduces to

$$
\frac{2 e^{2} r_{1}^{3}}{f^{5}} \text {. }
$$

Now we have seen that the function $\phi$ in the law of molecular attraction does not vary much with $z$ and $T$, in which case the attraction between two molecules varies approximately inversely as the fifth power of their distance of separation, and thus corresponds to the foregoing expression. But the resemblance goes further. Traube has shown ${ }^{1}$ that the apparent volume of an atom at the absolute zero is approximately proportional to the square root of its atomic weight, and hence $r_{1}^{3}$ is proportional to $\sqrt{a}$. Therefore if we make the assumption that the total electric charge of one sign associated with an atom is proportional to $\sqrt{a}$ (this is roughly the case) we obtain that the electric attraction between two atoms $c$ and $e$ is equal to

$$
\frac{k \sqrt{a_{c}} \sqrt{a_{e}},}{f^{5}}
$$

where $a_{c}$ and $a_{e}$ denote the atomic weights of the atoms, and $k$ a constant. The attraction between two molecules of the same kind composed of various atoms would accordingly be given by

$$
\frac{k\left(\Sigma \sqrt{a}^{2}\right.}{f^{5}} \text {. }
$$

This expression contains the fundamental factor $\Sigma \sqrt{a}$ contained in the law of attraction deduced from latent heat data.

The atom must however more properly be regarded in all cases as an assemblage of electrons and positive charges instead of a spherical con. ductor of electricity, whose effect on an external electron, or on another atom, is more or less equal to the total sum of the effect of each electrical charge. Now according to Rutherford ${ }^{2}$ the atom consists of a number of electrons located near the surface of the atom, and a corresponding positive charge concentrated more or less at the center. It is very probable that the electrons and corresponding positive charges of an atom are not arranged in a symetrical way with respect to the center of the atom. Under these conditions the atom would as a whole behave

1 Physik. Zeit., Oct., r909, p. 667.

2 Phil. Mag., 21, p. 669, I9II. 
approximately like an electrical doublet. Now it can be shown that the orientation of the axes of the atomic doublets would on the average be such as to produce attraction between the atoms. Thus suppose that the atoms are stationary in space and occupy their average positions, which correspond to the points of intersection of three sets of equidistant planes situated at right angles to each other. Suppose that the axes of the electrical doublets of a row of atoms lie on the straight line joining them, and that the charges are so arranged that opposite kinds face one another. Under these conditions the atoms will attract each other, tending to move along the straight line joining them. Let us also suppose that the atoms in an adjacent parallel row be similarly arranged except that the positive charge of each doublet faces in the opposite direction with respect to the negative charge, as shown in Fig. I. It will be evident

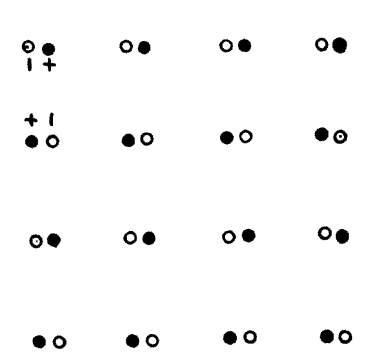

Fig. 1. that we may suppose that the set of atoms consists of parallel rows of the foregoing kind lying in parallel planes, and that in each alternate row of the rows lying in a plane the positive charges face one way, and in the other rows face in the opposite way. Hence we may also look upon the atoms as being divided up into a number of parallel rows at right angles to the foregoing rows, in which the charges of opposite sign will be nearer to each other than the charges of the same kind. Thus the atoms would also exert an attraction upon each other along the directions of these rows. Hence the atoms would exert an attraction upon each other in all directions, tending to contract the set of atoms as a whole.

In practice however, the atoms or molecules, are not stationary in space, but undergo motion of translation, which gives rise to collisions with each other, which have the effect of abruptly changing the orientations, or the directions of rotation, of the axes of the electrical doublets. But according to the principle that the potential energy of a system tends to become a minimum, the axes of the doublets would tend to point on the average in such directions as will give rise to an attraction between the atoms. A closer examination will show how this may be brought about. The axis of a doublet points successively in different directions in space through molecular collisions. Each direction will occur equally frequently since the substance as a whole has no electric polarity. But the successive directions of one atomic doublet are connected with the successive directions of an adjacent atomic doublet. For the axes of the doublets would tend to point parallel to each other, through the 
forces of attraction and repulsion which the charges of the doublets exert upon each other. Hence the axis of an atomic doublet would be oftener parallel to that of another atomic doublet than at right angles to it. This would give rise to attraction tending to be the outstanding force between the doublets. The extreme case of such a connection between the positions of the atomic doublets with respect to each other would be if the atomic doublets in Fig. I were to rotate round their centers in any manner but with their axes remaining parallel to each other.

It may happen, of course, that for certain densities of the substance the effect of molecular collision on the orientation of the axes of the electrical doublets may be such that repulsion is produced between the atoms instead of attraction. According to the foregoing principle connected with potential energy this repulsion would tend to be as small as possible. Thus atomic repulsion, whose existence is shown by the Joule Thomson effect, may be explained as well as attraction by the existence of electrical doublets.

In the case of two molecules the forces of attraction and repulsion would be due to the effect of the atomic doublets of one molecule on those of the other. The effect of each atomic doublet on another doublet would very probably be independent of the presence of the other doublets, and the electrical effect of a molecule would therefore in a sense be an additive quantity of its atoms.

It will be of interest next to examine the algebraical nature of the law of force according to the foregoing supposition. It can be easily shown that the force between two electrical doublets of magnitude $M^{\prime}$ and $M^{\prime \prime}$ is

$$
\frac{M^{\prime} M^{\prime \prime}}{r^{4}} 3\left\{2 \cos \cdot \theta \cdot \cos \theta^{\prime}-\sin \theta \cdot \sin \theta^{\prime}\right\}=\frac{M^{\prime} M^{\prime \prime}}{r^{4}} C_{e},
$$

where $r$ denotes the distance between the centers of the doublets and $\theta$ and $\theta^{\prime}$ the angles the doublets make with the line joining their centers. In the case in which the doublets belong to two atoms of a mon-atomic substance in the liquid or gaseous state the quantity $C_{e}$ would be a function of the two average angles the doublets make with the line joining them. These angles would evidently depend on the frequency of atomic collision in the substance, and on the nature of a collision, and hence would depend on the temperature and density of the substance, and on its nature, i.e., on its temperature, distance of atomic separation, atomic weight, and critical constants. In the case that the substance consists of molecules, $M^{\prime}$ would consist of the sum $\left(M_{1}{ }^{\prime}+M_{2}{ }^{\prime}+\cdots\right)$ of the atomic doublets of a molecule, and $M^{\prime \prime}$ of the sum $\left(M_{1}^{\prime \prime}+M_{2}{ }^{\prime \prime}+\cdots\right)$ 
of the doublets of another molecule. When repulsion exists, as we have seen may be the case, the sign of $C_{e}$ is negative.

The foregoing results are borne out by experiment. According to what has gone before the quantity $C_{e}$ would be proportional to

$$
\frac{\mathbf{I}}{z} \cdot \phi\left(\frac{T}{T}, \frac{z}{x_{c}}\right)
$$

and thus a function of the temperature of the substance, the distance of molecular separation, and the critical constants. It appears also that the moment of each electrical doublet is proportional to $\Sigma \sqrt{ } a$, the sum of the square roots of the atomic weights of the atoms. This result will be confirmed by other evidence later.

It will be of interest next to consider more closely the distribution of the electrons and positive charges in an atom. According to the experiments of Rutherford mentioned the positive charge is concentrated in a sphere of radius less than $10^{-12} \mathrm{~cm}$. From considerations of symmetry we would expect this charge to be the electrical center of the atom. Since the number of electrons is finite it is evident that they cannot be arranged around the positive charge in such a way that the electric force is zero on any point on a line passing through the positive charge. If the point selected is at a distance from the positive charge which is large in comparison with the distance of each electron, the effect is approximately equivalent to the sum of the effects of a number of doublets each of which corresponds to an electron in the atom, i.e., the atom as a whole behaves approximately like an electrical doublet. This corresponds to the state of affairs in practice, where the forces we are dealing with correspond to an average distance of separation of the atoms or molecules which is much larger than the atomic and molecular diameters. Of course, on rotating an atom round its center the moment of the average doublet will change somewhat, and we must therefore associate an average doublet with an atom which includes the consideration of direction from the atomic center. It is, of course, also not improbable that the positive charge is not located at the center of the atom.

If the two electrical charges of the representative doublet of an atom were separated by the same distance in all cases, each charge would be proportional to the square root of the atomic weight. But this is not likely to be the case. Moreover there is good reason to believe from various evidence that the number of unit positive charges in an atom is equal to $A$ its atomic number. Therefore if $x$ denote the distance of separation of the charges of the representative doublet of an atom, we have

$$
x A=\sqrt{x}
$$


Now the atomic number increases with the atomic weight, and for high atomic weights is approximately half of the atomic weight. Thus it appears that the distance $x$ decreases with the atomic weight of the atom. This is probably due to the fact that the larger the number of electrons in an atom, the nearer can their distribution be made symmetrical around the positive charge.

Since the nature of the quantity $C_{e}$ in the expression for the force of two atomic or molecular doublets upon each other, which corresponds to the function $(\phi)$ in the law of molecular attraction, depends on the average orientation of the atomic or molecular doublets with respect to each other in a substance, the function $(\phi)$ would depend, as has already been pointed out, on the nature of the atomic and molecular interaction. It should therefore be a function of the temperature and the density of the substance, as we have already seen is the case. In general, it will be evident that any process that will change the nature of the molecular interaction will change the nature of the function $(\phi)$.

Thus for example, in the case of a mixture of molecules $a$ and $c$ the nature of the interaction of a molecule $a$, or $c$, with other molecules would be different from that when the constituents are in the pure state. The attraction between two molecules $a$ would then be given by the general expression

$$
\psi\left(R_{a}{ }^{\prime}, \rho\right) \cdot(\Sigma \sqrt{a})^{2},
$$

where $R_{a}{ }^{\prime}$ denotes the ratio of the different constituents of the mixture, and $\rho$ its density. The nature of the function $\psi$ could evidently not be predicted from the form of $(\phi)$ applying to a pure substance. Similar remarks apply to the nature of the force between two molecules $a$ and $c$, or two molecules $c$. The nature of the function $\psi$ could be determined only from data relating to mixtures.

The nature of the molecular interaction of the molecules of a liquid would evidently be changed by the application of an electric field, since it would tend to set the doublets parallel to each other, and a corresponding change in the law of molecular attraction would be introduced. This might therefore be attended by a change in the volume of the substance. Since molecules and atoms possess magnetic properties a magnetic field would act in the same way.

The motion of a substance through space would tend to make the electrical atomic doublets set themselves with their axes at right angles to the motion, an effect which would become very marked when the velocity of the substance approaches that of light. This would obviously change the nature of molecular interaction, and accordingly a change in the functional nature of the law of molecular attraction be introduced. 
Hence on giving a motion to a liquid it would undergo a contraction or an expansion as the case may be. When the substance is a solid the change in length in the direction of the motion might be different from that at right angles to it.

Since light consists of electromagnetic waves, or of waves of electric and magnetic force, it would have the effect of changing the nature of molecular interaction to a certain extent and hence the nature of the forces of interaction. The chemical changes induced by light in liquids and gases could be well and strikingly explained in this way.

The Passage of the $\alpha$ Particle through Matter.

The behavior of a charged particle passing through matter is intimately connected with the foregoing considerations. Bragg and the writer ${ }^{1}$ have carried out some measurements on the range of the $\alpha$ particle in different gases, that is, the distance it traverses during which its energy decreases to a certain fraction of its original value. The $\alpha$ particle, it should be mentioned, is according to Rutherford a helium atom carrying a positive charge equal to $2 e$. It was found that the range is inversely proportional to the sum of the square roots of the atomic weights of the atoms of a molecule of the gas. The passage of an electron through matter (the electron carries a negative charge equal to $e$ ), one would expect, should be subject to the same law. The writer has obtained some evidence that this is the case for velocities of the electron of the same order as those of the $\alpha$ particle. ${ }^{2}$

Let us suppose that the $\alpha$ particle mainly spends its kinetic energy in increasing that of the molecules along its path by means of the forces of attraction and repulsion it exerts upon them. Now Maxwell has investigated the motion of a molecule that expends its energy in a gas in this way for the case when the molecule is attracted or repelled by a molecule of the gas according to a law of the form $K / z^{n}$, where $z$ denotes the distance of separation of the interacting molecules, and $K$ and $n$ are constants. $^{3}$ Thus the decrease of the velocity $V$ of a molecule of mass $m_{2}$ in passing over a distance $d x$ in a gas whose molecules are each of mass $m_{3}$, is according to this investigation given by

$$
\frac{d V}{d x}=\frac{-m_{3}}{m_{2}+m_{3}}\left(\frac{K\left(m_{2}+m_{3}\right)}{m_{2} m_{3}}\right)^{2 /(n-1)} N A_{c} V^{(n-5) /(n-1)},
$$

where $N$ denotes the number of molecules in the gas per cubic $\mathrm{cm}$., and $A_{c}$ a constant which is independent of $m_{2}, m_{3}$ and $V$. The force between

1 Phil. Mag., ro, 1905, p. 3 I8.

2 Proc. Roy. Soc., A, vol. 84, p. 20, I9Io.

${ }^{3}$ Conduction of Electricity through Gases, by J. J. Thomson, 2d ed., p. 37 I. 
a neutral gas molecule and a charged $\alpha$ particle, according to the preceding Article, corresponds to the force between the electrical doublet of the neutral molecule, and the electrical charge and the electrical doublet of the $\alpha$ particle. It will not be difficult to see that the force between the two doublets is small in comparison with that due to the doublet of the neutral molecule and the charge on the $\alpha$ particle, and hence the latter force only need be considered. It can easily be shown that if a charged particle is situated on the prolongation of the axis of a doublet the force between the two is $M 2 e / r^{3}$, where $M$ denotes the moment of the doublet, $2 e$ the charge on the particle, and $r$ their distance of separation. This force may be either positive or negative in sign as the case may be. The doublet of a molecule is however continually undergoing varying oscillations and rotations. The average force between a neutral molecule and the $\alpha$ particle during its motion through the gas, may thus be written $C_{e}{ }^{\prime} \cdot M / r^{3}$, where $C_{e}{ }^{\prime}$ is a constant. It might be conjectured at first sight that $C_{e}{ }^{\prime}$ is a function of the temperature and density of the gas. But this is evidently not the case since the various positions of the doublets of the neutral molecules with respect to the rapidly passing $\alpha$ particle must be symmetrically distributed in space because the gas as a whole has no polarity, and hence these positions are independent of the concentration and temperature of the molecules. The moment $M$ of the doublet of a molecule is according to the preceding Article proportional to the sum of the square roots of the atoms of a molecule, or proportional to $\Sigma \sqrt{a}$. The law of force between the $\alpha$ particle and a neutral molecule may accordingly be written $C_{x} \Sigma \sqrt{a} / z^{3}$, where $C_{x}$ is a constant. The above equation then reduces to

$$
\frac{d V}{d x}=\frac{C_{x}}{m_{2}} \Sigma \sqrt{a} \cdot \frac{N A_{e}}{V},
$$

which on multiplying by $m_{2} V \cdot d x$ and integrating becomes

$$
m_{2}\left(V_{1}^{2}-V_{2}^{2}\right)=C_{x}\left(x_{1}-x_{2}\right) \Sigma \sqrt{a}
$$

This equation states that an $\alpha$ particle whose energy falls from $\frac{1}{2} m_{2} V_{1}^{2}$ to $\frac{1}{2} m_{2} V_{2}^{2}$ traverses a distance $x_{1}-x_{2}$, which is inversely proportional to the square roots of the atomic weights of the atoms of a molecule of the gas. This is the law found by experiment, and which is thus in a striking way explained by the existence of electrical doublets in atoms and molecules.

It is interesting to note that according to the foregoing investigation the rate at which an $\alpha$ particle spends energy on a molecule is independent of the density and temperature of the gas through which the $\alpha$ particle 
passes. This is borne out by the experimental results. It also follows from the investigation that the $\alpha$ particle will cease spending its energy in the way deduced when it has lost its charge.

In the foregoing investigation it was assumed that the $\alpha$ particle spends its energy mainly in interacting with neutral molecules. This will appear highly probable when it is considered that less than ten molecules of ten thousand molecules lying in the path of the $\alpha$ particle are likely to get ionized.

PhySical LaBoratory, UNION COLLEGE,

Schenectady, N. Y., March 23, $192 \mathrm{I}$. 